\title{
Fast Parametric Estimation for Macroscopic Traffic Flow Model
}

\author{
Hassane ABOUAÏSSA* Michel FLIESS ${ }^{* *}$ Cédric JOIN ${ }^{* * *}$ \\ * LGI2A (E.A. 3926), Université d'Artois, \\ Technoparc Futura, 62400 Béthune, France \\ hassane.abouaissa@univ-artois.fr \\ ** INRIA-ALIEN \& LIX (CNRS, UMR 7161), École polytechnique, \\ 91128 Palaiseau, France \\ Michel.Fliess@polytechnique.edu \\ *** INRIA-ALIEN \&G CRAN (CNRS, UMR 7039), Nancy-Université, \\ BP 239, 54506 Vandœuvre-lès-Nancy, France \\ cedric.join@cran.uhp-nancy.fr
}

\begin{abstract}
The main traffic parameters such as the critical density and the free flow speed of a motorway segments are subject to changes over time due to traffic conditions (traffic composition, incidents, ...) and environmental factors (dense fog, strong wind, snow, ...). As such parameters have an impact on the performance of the traffic control strategies, they must be estimated on-line. Our approach, which is of algebraic flavor and avoids asymptotic and statistical techniques, yields fast implementable formulae in closed form. Some convincing computer simulations are provided. Copyright 2008 IFAC.
\end{abstract}

Keywords: Traffic control, linear systems, parameter identification.

\section{INTRODUCTION}

Intelligent transportation systems were introduced to ensure an optimal infrastructure utilization and to improve the driving safety, by alleviating congestion phenomena. All proposed control strategies rest on the use of models particularly the macroscopic one (Papageorgiou, HajSalem \& Blosseville (1991)). However, some of the important parameters of such a model are usually unknown and are subject to frequent changes over time (Ozbay, Ilgin \& Kachroo (2006)). The success of traffic control strategies depends therefore on the capacity to calibrate the control parameters as soon as one is able to track changes in the main traffic model characteristics (Wang \& Ioannou (2006)). The model parameters calibration is generally done off-line using simulation software as in Zhang, Boitor \& Ioannou (2005) or estimated off-line as in Karaaslan, Varaiya \& Walrand (1990). Nevertheless, as the parameters are subject to changes over time owing traffic conditions (traffic composition, incidents, ...) and environmental factors (dense fog, strong wind, snow, ...), their estimation must be achieved on-line. In this context, several parameter estimators are available. Bellemans, Schutter \& Moor (2002) have proposed a nonlinear optimization algorithm that refits the most sensitive traffic flow model parameters. The approach is based on a regular set of data. Whereas the method is very powerful, it requires more computation, and the fitting with one set of data may not guarantee a good results with another set of data. Ozbay, Ilgin \& Kachroo (2006) have used an extended Kalman filter (EKF) to estimate the critical density considered as state variable. Wang \& Ioannou (2006) have developed a parallel adaptive least-squares parametric estimator for a second-order macroscopic model. Several other works (see, e.g., Wang \& Papageorgiou (2005), Wang, Papageorgiou \& Messmer (2006)) are utilizing the same principle of EKF to estimate together traffic parameters and states. This paper ${ }^{1}$ is proposing a new fast parametric estimation method resulting from algebraic technics first introduced in Fliess \& Sira-Ramírez (2003, 2008) 2 . This new setting, which avoids statistical and asymptotic techniques, yields fast implementable algebraic formulae, in closed form. Excellent estimates of the most sensitive traffic flow model parameters, like the critical density and the free flow speed, are obtained in this way.

After recalling in Section 2 a basic macroscopic model, Section 3 demonstrates that the basic parameters may be estimated thanks to this new setting, which is briefly reviewed. Convincing numerical simulations ${ }^{3}$ are provided in Section 4.

\footnotetext{
1 See also Abouaïssa \& Join (2008). See Villagra, d'Andréa-Novel, Fliess \& Mounier (2008) for applications to another traffic control problem via analogous methods.

2 See Fliess, Join \& Sira-Ramírez (2004) for applications to linear diagnosis. See Fliess, Fuchshumer, Schöberl, Schlacher \& SiraRamírez (2008) for the corresponding parameter identification in the discrete-time case.

3 Lack of space is preventing us to detail the computer implementation. It may be obtained from $\mathrm{H}$. Abouaïssa (hassane.abouaissa@univ-artois.fr) and C. Join (cedric.join@cran.uhp-nancy.fr).
} 


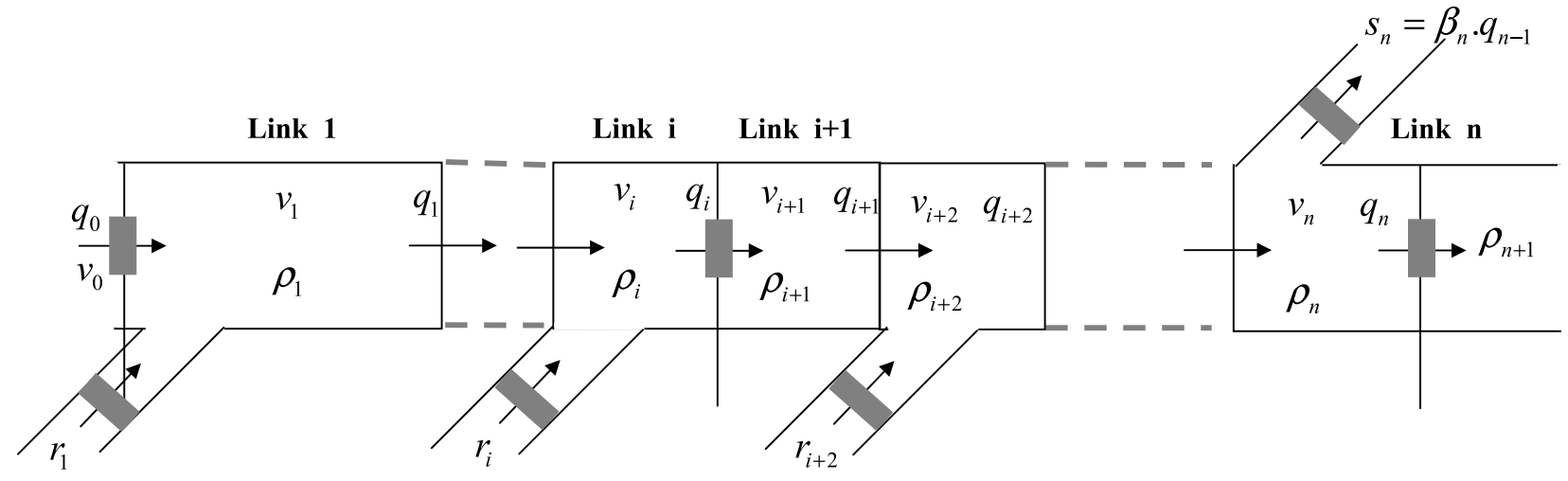

Fig. 1. A freeway stretch subdivided into segments

\section{TRAFFIC FLOW MODELLING}

We assume for the dynamics of the traffic flow a first order macroscopic model (LWR) (Lighthill \& Whitham (1955); Richards (1956)), which is briefly presented below. Consider a freeway stretch subdivided into $n$ segments as shown in Figure 1. The macroscopic first order model is based on the following mass conservation law:

$$
\frac{\partial \rho}{\partial t}+\frac{\partial q}{\partial x}=0
$$

For a space discrete representation, define the traffic density $\rho_{i}$ as the number of vehicles in segment $i$ at time $t$, divided by the segment length $L_{i}$. The traffic volume $q_{i-1}$ in $v e h / h$, is defined as the number of vehicles entering the segment $i ; q_{i}$ is the number of vehicles leaving the segment $i$. The equation of the nonlinear model for each freeway segment reads:

$$
\dot{\rho}_{i}(t)=\frac{1}{L_{i}}\left(q_{i-1}(t)-q_{i}(t)+a_{i} r_{i}(t)-b_{i} p_{i}(t)\right)
$$

where, $a_{i}$ and $b_{i}$ are binary variables which indicate respectively the presence or the absence of an on-ramp $r_{i}(t)$ and off-ramp $p_{i}(t)$. Note that another space discrete model (Mammar, Mammar \& Netto (2006)) that take into account the shock waves phenomena may also be utilized. Eq. (1) is supplemented by the following two equations:

$$
\begin{gathered}
q_{i}(t)=\rho_{i}(t) v_{i}(t) \\
v_{i}(t)=v_{f, i}\left(1-\frac{\rho_{i}(t)}{\rho_{m, i}}\right)
\end{gathered}
$$

Here, one considers the Greenshields fundamental diagram (Greenshields (1935)) based on Eq. (3), where, $v_{f, i}$ is the free flow speed or the speed of the vehicle in the segment $i$ when there are no interactions between vehicles. Write $\rho_{m, i}$ the jam density of the segment $i$. Note that each segment is characterized by a capacity defined by the maximum flow $q_{m, i}$ corresponding to the critical density $\rho_{c r, i}$. There is a linear relation between this density and the maximal density $\rho_{m, i}$ :

$$
\rho_{c r, i}=\frac{\rho_{m, i}}{2}
$$

As stated in Ozbay, Ilgin \& Kachroo (2006), the segment capacity is not constant. It may change over time owing to circumstances such as traffic condition and driver behavior, traffic composition and external factors (environmental conditions and segment geometry, ....). Strong wind and dense fog, for example, do also affect the flow-density and the speed-flow relationships. This means that both the critical density and the free flow speed are subject to changes, which may be quite abrupt. Due to their impact on the control strategies performances, these two main parameters ought to be estimated on-line.

\section{ON-LINE PARAMETER ESTIMATION}

Let us start with an elementary academic example for explaining the basic principles of our techniques (see Fliess \& Sira-Ramírez (2003, 2008) for details).

\subsection{An elementary academic example}

Consider the first-order monovariable input-output system

$$
\dot{y}(t)=K y(t)+u(t)
$$

for $t \geq 0$, where:

- $u(t)$ and $y(t)$ are respectively the control and output variables,

- the constant gain $K$ is unknown.

Rewrite Eq. (4) in the usual operational form (see, e.g., van der Pol \& Bremmer (1955); Yosida (1984)), which is most often introduced via the classic Laplace transform:

$$
s \hat{y}=K \hat{y}+\hat{u}+y(0)
$$

Apply to both sides the derivative $\frac{d}{d s}$, which corresponds in the time domain to the multiplication by $-t$ (van der Pol \& Bremmer (1955); Yosida (1984)), in order to eliminate the initial condition $y(0)$, the measurement of which might be corrupted by noises:

$$
\hat{y}+s \frac{d \hat{y}}{d s}=K \frac{d \hat{y}}{d s}+\frac{d \hat{u}}{d s}
$$

It yields the linear identifiability (Fliess \& Sira-Ramírez (2003, 2008)) of $K$ :

$$
K=\frac{\hat{y}+s \frac{d \hat{y}}{d s}-\frac{d \hat{u}}{d s}}{\frac{d \hat{y}}{d s}}
$$

In order to get rid of time derivatives, multiply both sides of Eq. (5) by $s^{-N}$, where $N \geq 1$ is large enough. Remember that negative powers of $s$ corresponds in the time-domain to iterated time-integrals (van der Pol \& Bremmer (1955); Yosida (1984)). Those iterated integrals are classic instances of low pass filters, which are attenuating corrupting noises, when considered as highly fluctuating phenomena ${ }^{4}$.

\footnotetext{
4 See Fliess (2006) for more details. Let us emphasize that no statistical tools are needed.
} 
Remark 3.1. The above calculations were for simplicity's sake assuming noises of zero mean. This is not the case in the numerical experiments of Section 4, where there is a constant mean equal to 150 . Let us indicate very briefly how to modify our previous calculations ${ }^{5}$. Replace Eq. (4) by $\dot{y}(t)=K y(t)+u(t)+C$ where $C$ is an unknown constant. Eq. (5) becomes $s \hat{y}=K \hat{y}+\hat{u}+y(0)+\frac{C}{s}$. We are eliminating $y(0)$ and $C$ by multiplying both sides of the last equation by the differential operator $\frac{d^{2}}{d s^{2}} s$. The remaining estimation of $K$ is quite similar to what we have already done.

Remark 3.2. An excellent estimated value of $K$, which is derived via iterated time integrals, may be obtained by utilizing quite short time windows (Fliess \& Sira-Ramírez $(2003,2008))$. This is why we may speak of a fast or on-line parametric estimation technique.

\subsection{Traffic flow parametric estimation}

The speed $v_{i}$ is easily obtained via Eq. (2) by utilizing the measured signals $q_{i}$ and $\rho_{i} \neq 0$. In what follows $v_{i}$ is thus supposed to be known.

Set $\theta_{1, i}=v_{f, i}$ and $\theta_{2, i}=\frac{v_{f, i}}{2 \rho_{c r, i}}$ for estimating the parameters $v_{f, i}$ and $\rho_{c r, i}$, which are linearly identifiable. Eq. (3) reads here:

$$
v_{i}(t)=\theta_{1, i}-\theta_{2, i} \rho_{i}(t)
$$

It becomes in the operational domain:

$$
\hat{v}_{i}(s)=\frac{\theta_{1, i}}{s}-\hat{\rho}_{i}(s) \theta_{2, i}
$$

if the unknown parameters $\theta_{1, i}$ and $\theta_{2, i}$ are assumed to be constant. Multiplying both sides of the preceding equation by $\Pi_{1}=\frac{1}{s^{2}} \frac{d}{d s} s$ and $\Pi_{2}=\frac{1}{s}$ yields:

$$
\begin{aligned}
& \frac{\hat{v}_{i}(s)}{s^{2}}+\frac{1}{s} \frac{d}{d s} \hat{v}_{i}(s)=-\theta_{2, i}\left(\frac{\hat{\rho}_{i}(s)}{s^{2}}+\frac{1}{s} \frac{d}{d s} \hat{\rho}_{i}(s)\right) \\
& \frac{\hat{v}_{i}(s)}{s}=\frac{\theta_{1, i}}{s^{2}}-\theta_{2, i} \frac{\hat{\rho}_{i}(s)}{s}
\end{aligned}
$$

where only iterated integrals appear in order to get a good noise attenuation. The well known Cauchy rule yields in the time-domain the following estimators:

$$
\begin{aligned}
{\left[\theta_{2, i}\right]_{e} } & =-\frac{\int_{0}^{T}(T-2 \tau) v_{i}(\tau) d \tau}{\int_{0}^{T}(T-2 \tau) \rho_{i}(\tau) d \tau} \\
{\left[\theta_{1, i}\right]_{e} } & =\frac{1}{T}\left(\left[\theta_{2, i}\right]_{e} \int_{0}^{T} \rho(\tau) d \tau+\int_{0}^{T} v_{i}(\tau) d \tau\right)
\end{aligned}
$$

where $[0, T]$ is the time window, which is necessary for achieving reliable calculations. This time window, which is quite short, permits on-line estimations of $\left[v_{f, i}\right]_{e}=\left[\theta_{1, i}\right]_{e}$ and $\left[\rho_{c r, i}\right]_{e}=\frac{\left[\theta_{1, i}\right]_{e}}{2\left[\theta_{2, i}\right]_{e}}$.

Remark 3.3. See, for instance, Fliess \& Sira-Ramírez (2003) when the denominators in the above formulae are close to zeros and yield numerical instabilities.

\footnotetext{
${ }^{5}$ See Fliess (2006) and Fliess \& Sira-Ramírez $(2003,2008)$ for more details.
}

\section{NUMERICAL EXPERIMENTS}

\subsection{First results}

Consider a simple section of $1 \mathrm{~km}$ length. The nominal traffic flow changes between 1800 and $1400 \mathrm{veh} / \mathrm{h}$. Set

$$
\left\{\begin{array}{l}
v_{f}=60 \mathrm{~km} / \mathrm{h} \text { for } t<0.4 \mathrm{~h} \\
v_{f}=72 \mathrm{~km} / \mathrm{h} \text { for } t \geq 0.4 \mathrm{~h}
\end{array}\right.
$$

and

$$
\left\{\begin{array}{l}
\rho_{c r}=60 \mathrm{veh} / \mathrm{km} \text { for } t<0.7 \mathrm{~h} \\
\rho_{c r}=48 / \mathrm{km} \text { for } t \geq 0.7 \mathrm{~h}
\end{array}\right.
$$

Figure 2 demonstrates that, without corrupting noises, good estimates are obtained almost immediately after the parameter variations. A very small integration time window, here 10 samples $^{6}$, is sufficient.

The output measurements $q_{i n}, \rho$ are now corrupted by an additive non-Gaussian noise, of mean 150, which is fluctuating between 0 and 300. Without pre-filtering ${ }^{7}$, this noise is degrading the reconstruction of $v$. Compare Figures 2-(c) and 3-(c). We have to increase the integration time window from $10 T_{e}$ to $600 T_{e}$, in order to ensure good and robust estimates.

\subsection{Application to a ramp metering}

The equation for the same section as above, with an onramp, reads:

$$
\dot{\rho}(t)=\frac{1}{L}\left(q_{\text {in }}(t)-q_{\text {out }}(t)+r(t)\right)
$$

where $L$ and $r(t)$ are the length and the control variable. Combining it with Eq. (2), (3) yields:

$$
\dot{\rho}(t)=\frac{1}{L}\left(q_{i n}(t)-\rho(t)\left[v_{f}\right]_{e}\left(1-\frac{\rho(t)}{2\left[\rho_{c r}\right]_{e}}\right)+r(t)\right)
$$

where $[\bullet]_{e}$ denotes the estimated value. The objective of the control, is to maintain the traffic density below a critical one $\rho<\rho_{c r}$. Let us define the maximal density $\rho_{m}=120 \mathrm{veh} / \mathrm{km}$. For a free-flow speed of $v_{f}=60 \mathrm{~km} / \mathrm{h}$ and a critical density $\rho_{c r}=60$, the section capacity expressed in term of traffic flow $q_{m}=1800 \mathrm{veh} / \mathrm{h}$. The initial density $\rho(0)=10 \mathrm{veh} / \mathrm{km}$, which means that the traffic is in an un-congested regime.

As already noticed by Abouaïssa, Iordanova \& Jolly (2007), Eq. (6) defines a controllable linear system which is therefore flat (see Fliess, Lévine, Martin \& Rouchon (1995); Fliess \& Marquez (2000); Rotella \& Zambettakis (2007); Sira-Ramírez \& Agrawal (2004)); $y=\rho$ is a flat output. It yields the closed-loop control

$$
r(t)=L \dot{y}^{*}-K_{1}\left(y-y^{*}\right)+y\left[v_{f}\right]_{e}\left(1-\frac{y}{2\left[\rho_{c r}\right]_{e}}\right)-q_{\text {in }}
$$

where the gain $K_{1}$ is to be tuned. We do not consider the queue length that can be formed at the on-ramp and may provide negative ramp flow values. The results reported in Figure 4 confirm the adaptive capabilities for tracking any change of the reference density. To avoid abrupt changes of the reference, $\left[\rho_{c r}\right]_{e}$ and $\left[v_{f}\right]_{e}$ are filtered by a low pass filter and becomes $\left[\rho_{c r}\right]_{f}$ and $\left[v_{f}\right]_{f}$.

\footnotetext{
6 The sampling period $T_{e}$ is equal to $1 \mathrm{~s}$.

7 Filtering those two measurements would increase the estimation performances. We did not do it here in order to test our approach.
} 


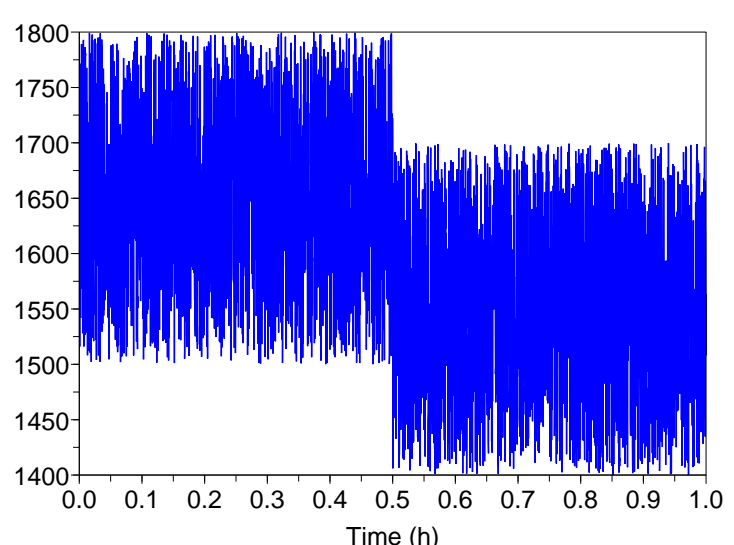

(a) $q_{\text {in }}$

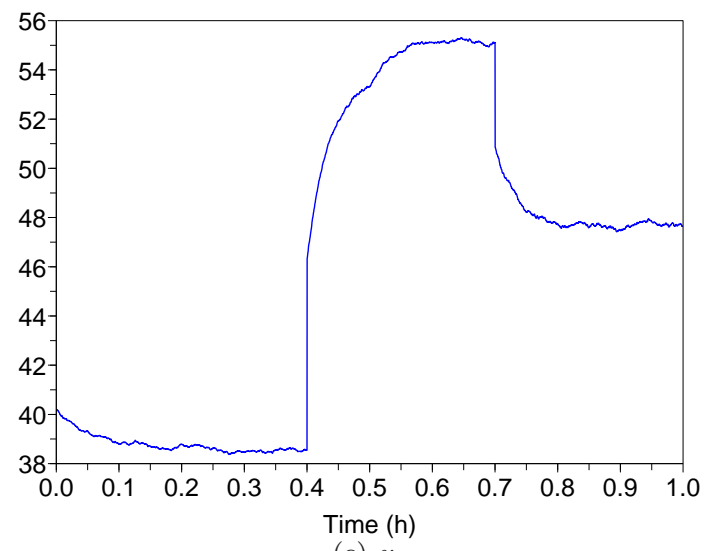

(c) $v$

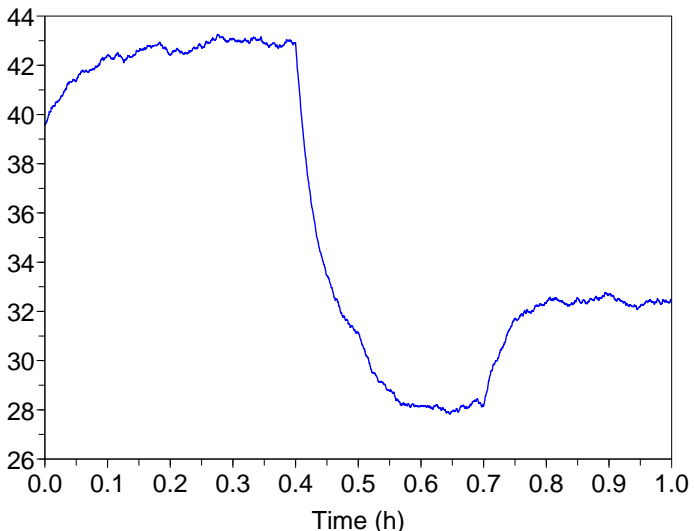

(b) $\rho$

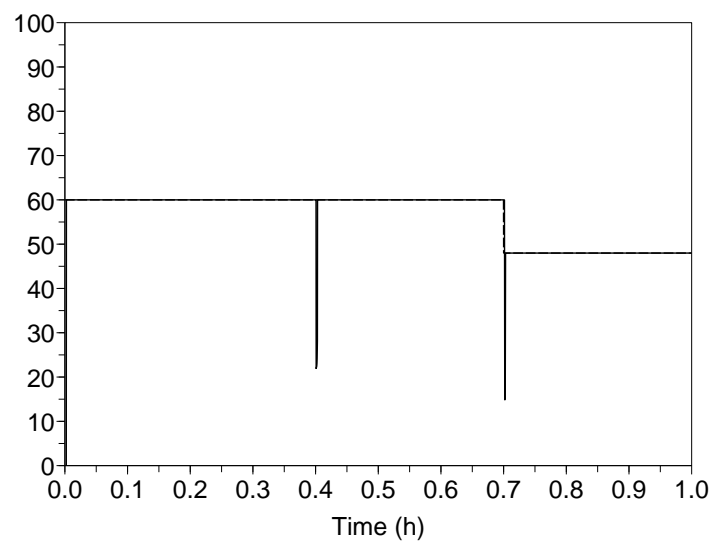

(d) $\left[\rho_{c r}\right]_{e}($ black -$), \rho_{c r}$ (black - -)

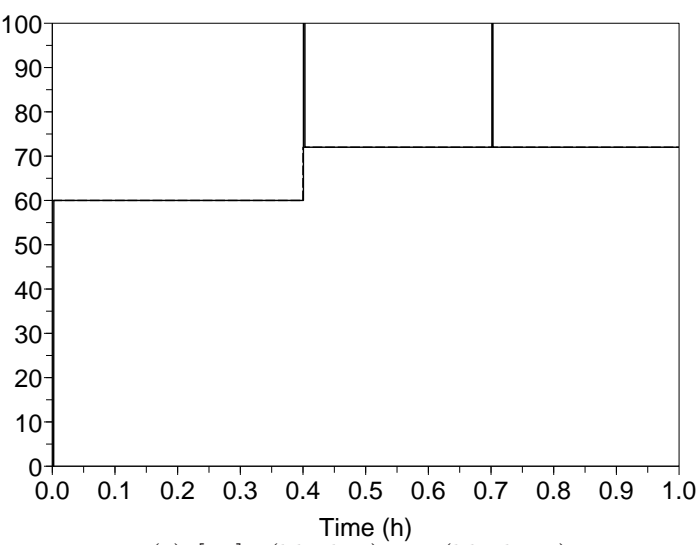

(e) $\left[v_{f}\right]_{e}\left(\right.$ black -), $v_{f}$ (black - -)

Fig. 2. Simulation results with noise-free measurements 


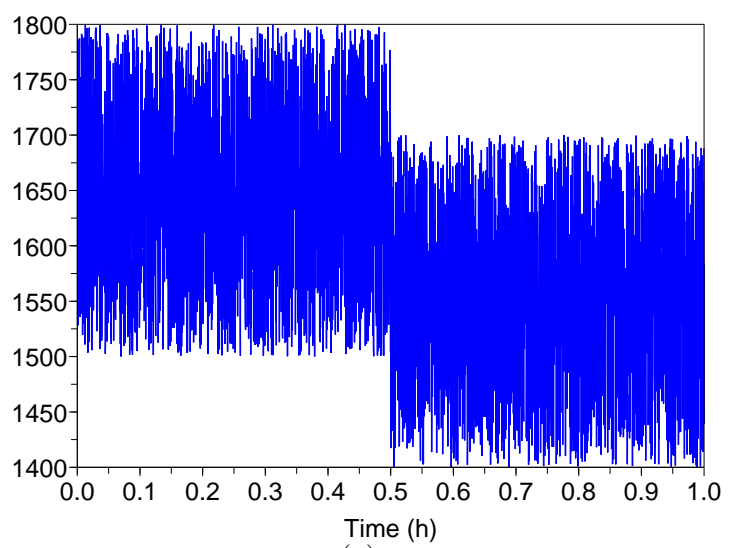

(a) $q_{\text {in }}$

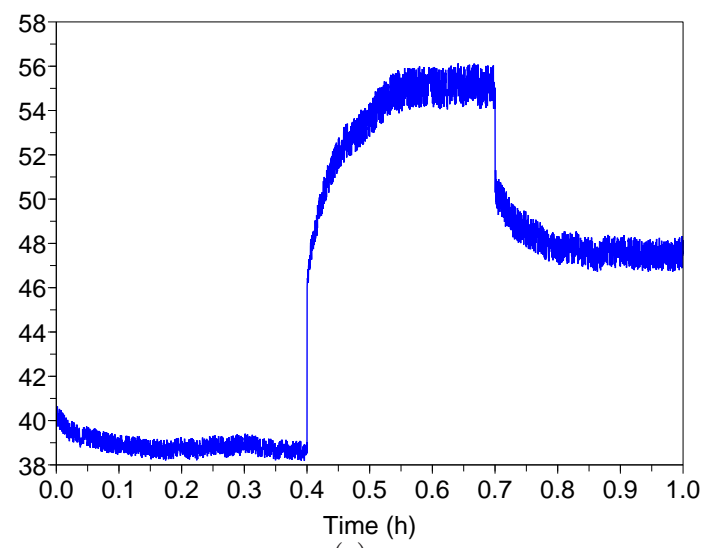

(c) $v$

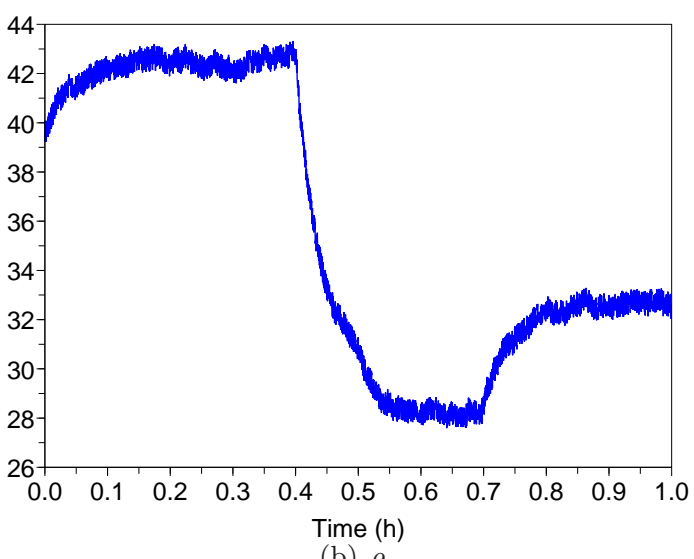

(b) $\rho$

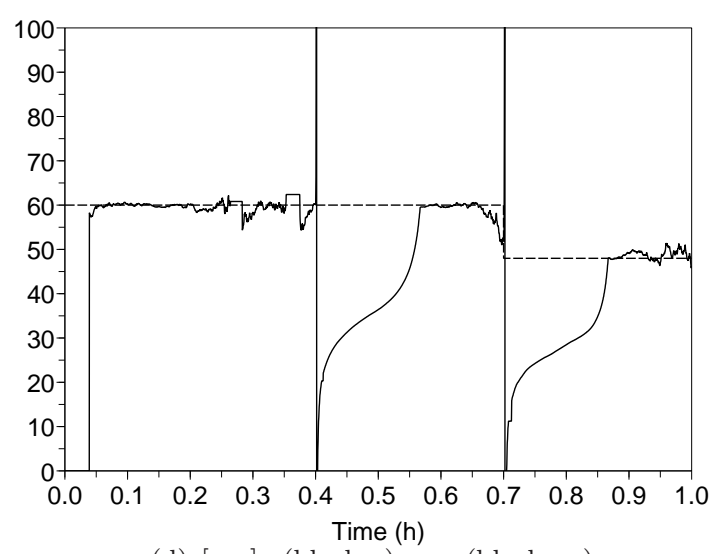

(d) $\left[\rho_{c r}\right]_{e}($ black -$), \rho_{c r}$ (black - -)

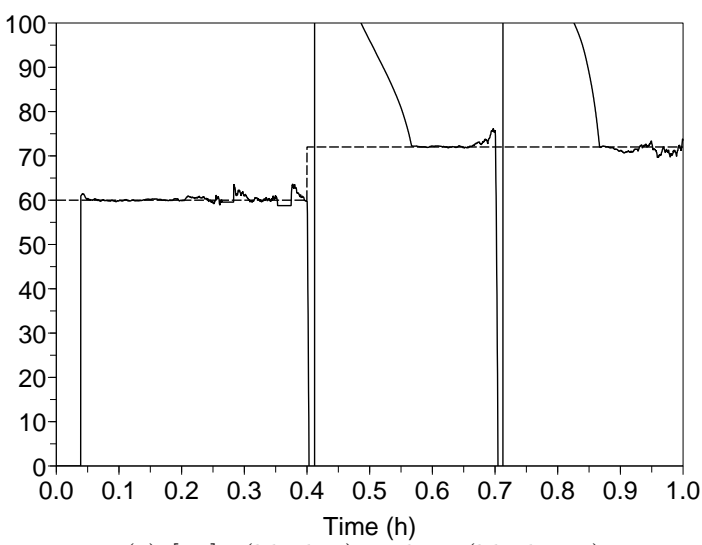

(e) $\left[v_{f}\right]_{e}\left(\right.$ black -) and $v_{f}$ (black - - )

Fig. 3. Simulation results in the noisy case 


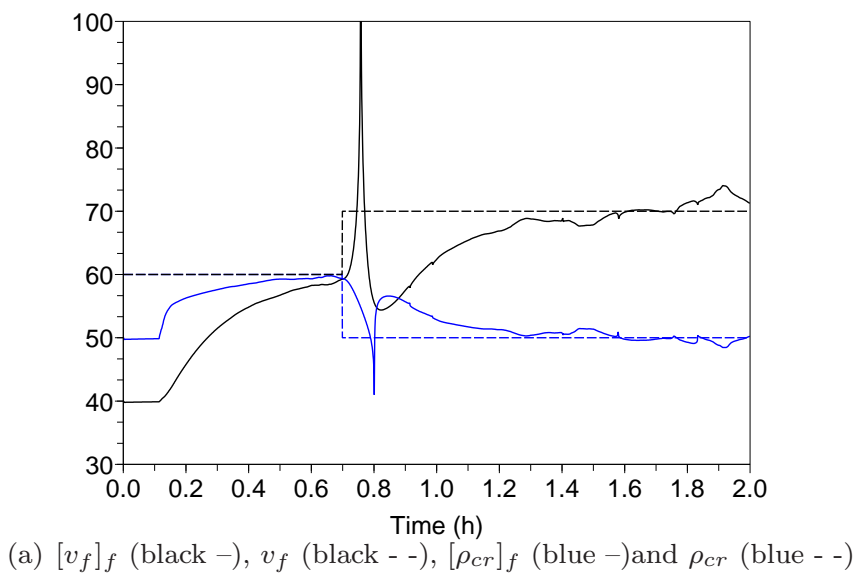

Fig. 4. Simulations in closed-loop, with noisy measurements

\section{CONCLUSION}

Future works will present more realistic scenarios for traffic control. The new on-line nonlinear estimation and identification techniques (Fliess, Join \& Sira-Ramírez (2008)) should play an important role.

\section{REFERENCES}

H. Abouaïssa, V. Iordanova and D. Jolly. Integrated control of highway systems using flatness-based concept, Proc. SCS-IFAC, Int. Modeling Simulation Multiconf. - Int. Conf. Advances Vehicle Control and Safety, Buenos Aires, 2007.

H. Abouaïssa and C. Join. Modélisation macroscopique du flux de trafic basé sur l'estimation paramétrique des modèles bond graphs, Actes re Conf. Int. Modélisation Simulation - MOSIM'08, Paris, 2008 (available at http://hal.inria.fr/inria-00250900/en/).

T. Bellemans, B.D. Schutter and B.D. Moor. Model predictive control with repeated model fitting for ramp metering. Proc. $5^{\text {th }}$ Int. IEEE Conf. Intelligent Transportation Systems, Singapore, 2002 .

M. Fliess. Analyse non standard du bruit, C.R. Acad. Sci. Paris, ser. I, vol. 342, pp. 797-802. 2006.

M. Fliess, S. Fuchshumer, M. Schöberl, K. Schlacher and H. Sira-Ramírez. An introduction to algebraic discretetime linear parametric identification with a concrete application, J. europ. syst. automat., 2008 (available at http://hal.inria.fr/inria-00188435/en/).

M. Fliess, C. Join and H. Sira-Ramírez. Robust residual generation for linear fault diagnosis: An algebraic setting with examples. Int. J. Control,, vol. 77, pp. 1223-1242, 2004.

M. Fliess, C. Join and H. Sira-Ramírez. Non-linear estimation is easy, Int. J. Modelling Identification Control, vol. 3, 2008 (available at http://hal.inria.fr/inria-00158855/en/).

M. Fliess, J. Lévine, P. Martin and P. Rouchon. Flatness and defect of non-linear systems: Introductory theory and examples. Int. J. Control, vol. 61, pp. 1327-1361. 1995.

M. Fliess and R. Marquez. Continuous-time linear predictive control and flatness: A module-theoretic setting with examples. Int. J. Control, vol. 73, pp. 606-623, 2000.

M. Fliess and H. Sira-Ramírez. An algebraic framework for linear identification. ESAIM Control Optimiz. Calculus Variat., vol. 9, pp. 151-168. 2003.

M. Fliess and H. Sira-Ramírez. Closed-loop parametric identification for continuous-time linear systems via new algebraic techniques, in H. Garnier \& L. Wang (Eds.): Continuous-Time Model Identification from Sampled Data., Springer 2008 (available at http://hal.inria.fr/inria-00114958/en/).

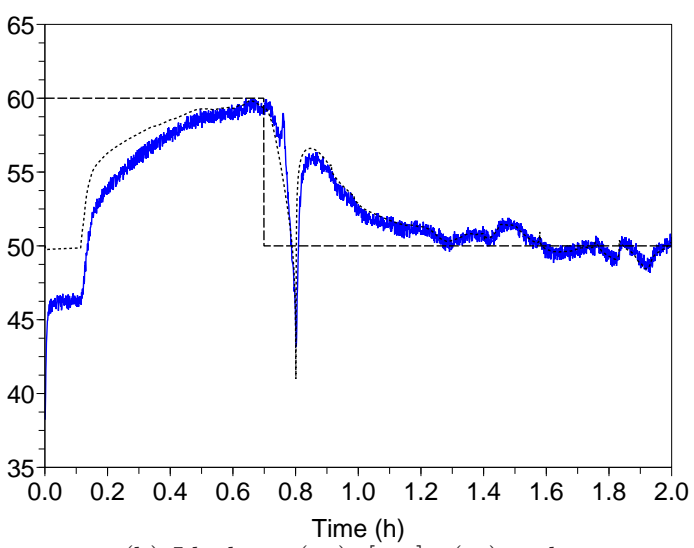

(b) Ideal $\rho_{c r}(--),\left[\rho_{c r}\right]_{f}(.$.$) and \rho$

B.D. Greenshields. A study of traffic capacity, Highway Research Board, vol. 14, pp. 448-477, 1935.

U. Karaaslan, P. Varaiya, and J. Walrand. Two proposals to improve freeway traffic flow, California PATH Research Report, Tech. Rep. UCB-ITS-PRR-90-6, 1990.

M.J. Lighthill and G.B. Whitham. On kinematic waves II: A theory of traffic flow in long crowded roads, Proc. Royal Soc. London, vol. A-229, pp. 317-345, 1955.

S. Mammar, S. Mammar and M. Netto. Coordinated ramp metering via second order sliding mode control. Proc. $9^{\text {th }}$ IEEE Intelligent Transportation Systems Conf. pp. 261-266. Toronto, 2006.

K. Ozbay, Y. Ilgin and P. Kachroo. Improved online estimation methods for a feedback-based freeway ramp metering strategy, Proc. $9^{\text {th }}$ IEEE Intelligent Transportation Systems Conf., pp. 412-417, Toronto, 2006.

M. Papageorgiou, H. Hadj-Salem, and J.M. Blosseville. Alinea: A local feedback control law for on-ramp metering, Transportation Research Record, vol. 1320, pp. 58-64, TRB, National Research Council, Washington D.C. 1991.

B. van der Pol and H. Bremmer. Operational calculus based on the two-sided Laplace integral, $2^{\text {nd }}$ ed., Cambridge University Press, 1955.

P.I. Richards. Shock waves on the highway, Operations Research, vol. 4, pp. 42-51, 1956.

F. Rotella and I. Zambettakis. Commande des systèmes par platitude, Techniques de l'ingénieur, S7450, 2007.

H. Sira-Ramírez and S.K. Agrawal. Differentially Flat Systems. Marcel Dekker, 2004.

J. Villagra, B. d'Andréa-Novel, M. Fliess and H. Mounier. Estimation of longitudinal and lateral vehicle velocities: An algebraic approach, Proc. Amer. Contr. Conf. - ACC'08, Seattle, 2008.

Y. Wang and M. Papageorgiou. Real-time freeway traffic state estimation based on extended kalman filter: a general approach, Transportation Research, vol. 39B, pp. 141-167, 2005.

Y. Wang, M. Papageorgiou, and A. Messmer. A real-time freeway network traffic surveillance tool, IEEE Trans. Control Systems Technology, vol. 14, pp. 18-32, 2006.

Y. Wang and P.A. Ioannou. Real-time parallel parameter estimators for a second-order macroscopic traffic flow model Proc. $9^{\text {th }}$ IEEE Intelligent Transportation Systems Conf., pp. 1466-1470, Toronto, 2006

K. Yosida. Operational Calculus, Springer, 1984.

J. Zhang, A. Boitor and P.A. Ioannou. Design and evaluation of roadway controller for freeway traffic, Proc. $8^{\text {th }}$ IEEE Intelligent Transportation Systems Conf., Vienna, 2005. 